\title{
BMJ Open Programmes to prepare siblings for future roles to support their brother or sister with a neurodisability: protocol of a scoping review
}

\author{
Linda Nguyen (D) , ${ }^{1}$ Jael Bootsma (D) , ${ }^{1}$ Briano Di Rezze (D) , ${ }^{1}$ Susan Jack (D) , \\ Marjolijn Ketelaar (10) ,3,4 Jan Willem Gorter (1) ${ }^{5}$
}

To cite: Nguyen L, Bootsma J, Di Rezze B, et al. Programmes to prepare siblings for future roles to support their brother or sister with a neurodisability: protocol of a scoping review. BMJ Open 2021;11:e053184. doi:10.1136/ bmjopen-2021-053184

- Prepublication history and additional online supplemental material for this paper are available online. To view these files, please visit the journal online. (http://dx.doi.org/10. 1136/bmjopen-2021-053184).

Received 08 May 2021 Accepted 23 June 2021

Check for updates

(C) Author(s) (or their employer(s)) 2021. Re-use permitted under CC BY-NC. No commercial re-use. See rights and permissions. Published by BMJ.

For numbered affiliations see end of article.

Correspondence to

Linda Nguyen;

nguyel7@mcmaster.ca

\section{ABSTRACT}

Introduction Siblings share a lifelong bond in their relationship, and they may choose to provide support to their brother or sister with a neurodisability. Previous reviews summarised programmes that only focused on the behavioural, emotional and psychological outcomes of the siblings. There is a need to synthesise existing evidence and enhance our understanding about programmes for siblings to acquire knowledge, develop skills and become empowered that can help them to provide support to their brother or sister with a neurodisability. The objective of this review is to identify and map the characteristics and outcomes of programmes designed to prepare siblings in their future roles to support their brother or sister with a neurodisability.

Methods and analysis This review will be conducted using the Joanna Briggs Institute methodology for scoping reviews. An integrated knowledge translation approach will be used by partnering with the Sibling Youth Advisory Council comprised of siblings of individuals with a disability throughout all review phases. Databases to be searched include PsycINF0, Cumulative Index of Nursing and Allied Health Literature, Sociological Abstracts, Education Resources Information Center, EMBASE, Web of Science, MEDLINE (Ovid) and SPORTDiscus, from date of inception to November 2020. Studies of programmes designed for siblings of individuals with neurodisabilities, with no exclusion on the age of siblings or context, and published in English will be included. Extracted data will include details of programme structure and content, eligibility criteria and participants, context, study methods and outcomes. A summary of the results will be presented in a tabular form to provide an overview of the programmes with an accompanying narrative summary to address the research questions of this review.

Dissemination Findings from this review will be shared using dissemination strategies in partnership with the Sibling Youth Advisory Council. We will share the findings with key stakeholders such as healthcare providers, researchers, and patient and family advocacy groups.

\section{INTRODUCTION}

There is an estimated 150 million children and youth under the age of 18 with a disability, including a neurodisability. ${ }^{1}$ Neurodisability
Strengths and limitations of this study

- This review will use a systematic approach to synthesise information about programmes designed to support siblings in their future roles to individuals with a neurodisability across the lifespan.

- Findings from this review will be limited to siblings of individuals with a neurodisability, and may not be generalisable for other diagnoses.

- Sibling partners will be involved in all phases of the scoping review, to ensure that the findings of this review are relevant and meaningful for stakeholders.

can be broadly defined as a group of congenital or acquired long-term conditions due to an impairment of the brain and/or neuromuscular system that may create functional limitations. $^{2}$ Children and youth with a neurodisability are growing up and becoming adults. During their transition to adulthood, they may take on different opportunities to explore their interests and goals, including school, work, family and leisure. They might also experience biological, social and emotional challenges, such as exploring the option to attend postsecondary education, finding employment, developing long-term relationships and navigating adult healthcare services. ${ }^{34}$ Even as adults, they may continue to experience challenges with navigating different systems such as healthcare, education and social services. To navigate these different systems, individuals with neurodisabilities can turn to their families for support during the progression through emerging adulthood. ${ }^{4}$ Adults with neurodisabilities will continue to require support, such as with personal care and activities of daily living, as they age. ${ }^{56}$ Families are often in a position to provide the most optimal support because they have been involved throughout their child's care, in which they know their child 
best including the child's strengths, areas of improvement and goals. ${ }^{7}$

Siblings are an integral part of the family, and every sibling relationship is different with varying levels of emotional closeness, social connectedness and expectations of each other. ${ }^{8}$ Siblings have a unique relationship, in which they share a lifelong bond. When a brother or sister has a neurodisability, a sibling may choose to provide support. Yet, sibling relationships are also highly dynamic and can change over time depending on the needs, roles, and commitments of the whole family. ${ }^{9}$ Siblings may choose to support their family in different ways, such as providing emotional support to their brother or sister with a neurodisability or taking on responsibilities to help the whole family. ${ }^{10}$ Siblings often become closer when there is planning involved from the whole family with the recognition of the sibling's role for the future during adulthood. ${ }^{11} 12$ Sometimes, there is an implicit expectation that the siblings will eventually become carers for their brother or sister with a disability when parents are no longer able to provide support. ${ }^{13}$ Some siblings may find that clear and explicit expectations about future responsibilities can be helpful to understand their role. ${ }^{13}$ Having a sibling with a neurodisability could affect siblings' own future planning, such as career choice, partner choice or decision to have children. ${ }^{13}$ Many siblings will continue to be a part of the lives of their brother or sister with a neurodisability throughout the lifespan.

Siblings can offer support in different ways to their brother or sister with a neurodisability. There are four main types of support ${ }^{14}$ : concrete support that refers to acts of practical assistance; emotional support that includes acts of empathy; advice support that comprises of acts to offer information, emotional reassurance and guidance; and esteem support that focuses on the reinforcement of personal worth of an individual. Different programmes can help to prepare siblings for future roles to support their brother or sister with a neurodisability. These programmes can serve different purposes such as sharing information about neurodisabilities, providing opportunities for siblings to connect with each other and offering resources for the siblings to support their brother or sister with a disability. ${ }^{1516}$ The programmes can be tailored for different age groups. For example, Sibshops have been developed in the United States for siblings ages 8-13 years old to learn strategies to deal with situations experienced by their brother or sister with a disability. ${ }^{15}$ Another programme is Sibs Talk, which was developed in the UK for students to develop coping strategies, acquire knowledge about their siblings' disability, address challenges about their experiences at home and school, and identify their responsibilities to their siblings with a disability. ${ }^{17}$ Family interventions may also include sessions for siblings to learn about the disability of their brother or sister ${ }^{18}$ or learn strategies to interact and socialise with their brother or sister that is reinforced by parents. ${ }^{19}$ During adulthood, siblings of individuals with a neurodisability might continue to seek supports and resources to address their concerns, such as the mental health of their whole family, housing options and finances. ${ }^{20}$

Three systematic reviews have explored interventions and programmes for siblings of a brother or sister with a disability. ${ }^{21-23}$ Hartling et al evaluated the effectiveness of sibling programmes that focused on improving behavioural and emotional outcomes in siblings of a brother or sister with a disability and discussed the need for programmes to clearly describe the purpose and intended benefits. Tudor and Lerner provided a review on the nature, content and outcomes of clinical services designed for siblings of a brother or sister with a developmental disability, and identified how future research can be conducted to identify which siblings might benefit from certain services. McKenzie Smith et al conducted a systematic review to summarise the evidence about the psychological functioning of siblings of a brother or sister with a chronic physical or mental health condition. All three systematic reviews summarised the literature about sibling programmes that focused on the behavioural, emotional and psychological outcomes of the siblings themselves. This review will focus on programmes designed to prepare siblings in their roles so that they can, in turn, support their brother or sister with a disability. There is a need to synthesise the literature on sibling programmes that focus on the knowledge acquisition, skill development, and empowerment of siblings of individuals with a neurodisability.

The aim of this scoping review is to identify and map the characteristics and outcomes for participants in programmes designed to prepare siblings in their future roles to support their brother or sister with a neurodisability. A preliminary search on the Joanna Briggs Institute (JBI) Database of Systematic Reviews and Implementation Reports, Cochrane Database of Systematic Reviews, PROSPERO, PubMed and Cumulative Index of Nursing and Allied Health Literature (CINAHL) did not identify any reviews that have summarised the literature about this topic. The results from this review will provide an understanding about existing programmes designed for siblings to support their brother or sister with a neurodisability. Results will highlight information about the content of these programmes of how siblings can provide support to their brother or sister with a neurodisability. We plan to use key findings from this review to identify current practices, as well as inform the development of resources and tools to support siblings of individuals with neurodisabilities in cocreation with key stakeholders, such as siblings of individuals with neurodisabilities and researchers. Based on recommendations from the UK Medical Research Council, available evidence should be used to inform the development of a resource or intervention. ${ }^{24}$

\section{Review questions}

Our primary review questions are:

i. What are the characteristics of programmes (eg, purpose, description, eligibility criteria, length, activities, service provider, delivery)? 
ii. What are the outcomes for the siblings of individuals with neurodisabilities participating in the 2 programmes?

\section{METHODS}

This scoping review will be conducted according to the JBI methodology for scoping reviews. ${ }^{25}$ This protocol paper, as well as the final report will be written using the Preferred Reporting Items for Systematic Reviews and Meta-Analyses extension for Scoping Reviews (PRISMA-ScR) checklist. ${ }^{26}$

\section{Patient and public involvement}

This scoping review also uses an integrated knowledge translation as an approach to doing research with knowledge users as equal partners with researchers throughout the study. ${ }^{27}$ In this review, we partnered with the Sibling Youth Advisory Council (SibYAC) comprised of six young adult siblings who have a brother or sister with a disability. Based on previous literature, siblings with the lived experiences of having a brother or sister with a disability have often described the importance of being involved in research. ${ }^{28}$ The SibYAC identified the relevance of the research questions for this review and programme outcomes (eg, knowledge acquisition, skill development, empowerment) that are important to synthesise in this review. We plan to continue our partnership with the SibYAC throughout the process of conducting this review, such as reviewing preliminary findings and providing recommendations for the interpretation of results, as well as knowledge translation stages such as cocreating reports and presentations or sharing the results with the community.

\section{Search strategy}

A three-step search strategy will be employed. An initial limited search of PsycINFO was undertaken on 17 November 2020 to identify articles on the topic. The text words contained in the titles and abstracts of relevant articles, and the index terms used to describe the articles were used to develop a full search strategy for PsycINFO (see online supplemental file 1). The search strategy, including all identified keywords and index terms, will be adapted for each included database and/or information source. The reference list of all included sources of evidence will be screened for additional studies. Studies published in English will be included. Articles published from database inception to the present will be included.

\section{Information sources}

The databases to be searched include PsycINFO, CINAHL, Sociological Abstracts, Education Resources Information Center, EMBASE, Web of Science, MEDLINE (Ovid) and Sport Discus.

\section{Study of evidence selection}

Following the search, all identified citations will be collated and uploaded into Covidence, systematic review software
(Veritas Health Information, Melbourne, Australia) and duplicates will be removed. Following a pilot test, titles and abstracts will then be screened independently by two reviewers (LN and JB) against the inclusion criteria for the review. Potentially relevant sources will be retrieved in full with citation details. The full text of selected studies will be assessed in detail against the inclusion criteria by two independent reviewers (LN and JB). Reasons for exclusion of sources of evidence at full text that do not meet the inclusion criteria will be recorded and reported in the scoping review. Any disagreements that arise between the reviewers at each stage of the selection process will be resolved through discussion or consultation with a third reviewer. The results of the search and the study inclusion process will be reported in full in the final scoping review and presented in a PRISMA-ScR) flow diagram. ${ }^{29}$

\section{Inclusion criteria \\ Participants}

This review will focus on programmes with participants who are siblings of an individual with a neurodisability, defined as a group of congenital or acquired long-term conditions due to an impairment of the brain and/ or neuromuscular system that may create functional limitations. ${ }^{2}$ Sibling participants may be of varying ages, including children, youth and adults.

\section{Concept}

This review will include studies that describe programmes designed to support siblings in their roles. Outcomes of these programmes could include, but are not limited to knowledge acquisition, skill development, or empowerment for the siblings. Studies about sibling programmes that focus only on therapy or support for the siblings without reference to support the individual with a neurodisability will be excluded. Studies that describe sibling programmes without specific objectives targeting siblings of individuals with neurodisabilities will also be excluded.

\section{Context}

The context of this review will include all settings that deliver sibling programmes, such as school, rehabilitation, healthcare or community settings, in any country.

\section{Types of sources}

This review will consider all study designs including experimental and quasi-experimental study designs including randomised controlled trials, non-randomised controlled trials, before-and-after studies and interrupted time-series studies. Descriptive studies (eg, case reports), analytical observational studies (eg, prospective and retrospective studies, case-control studies and cross-sectional studies) will be included. This review will also consider descriptive observational study designs including case series, individual case reports and descriptive cross-sectional studies for inclusion. Qualitative studies will also be considered that focus on qualitative data including, but not limited to, designs such as phenomenology, grounded theory, ethnography, qualitative description, action research and 
feminist research. Mixed-methods studies will also be considered.

\section{Data extraction}

Data extraction from papers included in the scoping review will be performed independently by two reviewers (LN and JB) using a data extraction tool developed by all research team members, including the SibYAC. Data extracted will include the following: author(s), year of publication, country of origin (where the study programme was published or conducted), study aim(s), purpose(s) of the programme, study population and sample size, methodology/methods, programme context/setting, programme description, programme development (who developed the programme, process of developing the programme), programme delivery (including the provider and type of delivery), duration of the programme, programme activities, participants' needs and goals while participating in the programme, participant outcomes and programme evaluation. A draft of the data extraction sheet is provided in online supplemental file 2. This draft form was created based on the JBI template source of evidence details, characteristics and results extraction instrument (22), as well as the Cochrane Collaboration data extraction template (29). The data extraction sheet will be modified as necessary during the process of extracting data from each included evidence source. Modifications will be detailed in the scoping review. Any disagreements that arise between the reviewers will be resolved through discussion or consultation with a third reviewer. The authors of included articles will be contacted to request missing or additional data based on the data extraction sheet.

\section{Data analysis and presentation}

The extracted data will be presented in a tabular form that provides a comprehensive overview about sibling programmes based on the information outlined in the data extraction form. There will be an accompanying narrative summary to describe how the results address the research questions of this scoping review.

Overall, siblings can offer support in different ways as they will continue to be a part of their lives to their brother or sister with a neurodisability throughout the lifespan. While siblings may want to offer support, they need to be prepared and empowered to take on this role in the future. This scoping review addresses a gap in the literature about the available evidence about programmes designed to support siblings of individuals with a neurodisability in their future roles. The SibYAC will be involved as partners throughout the process of conducting this review to ensure that the findings are relevant and meaningful to the community. Findings from this review will provide a synthesis of evidence-based information about programmes as well as identify future directions to inform or enhance existing resources designed to support siblings of individuals with a neurodisability.

\section{DISSEMINATION}

The findings from this review will be published in peerreviewed publications, and presented at local, national and international conferences. We also plan to share a plain language report with the community. To support our knowledge translation and dissemination activities, we will leverage the infrastructure of a website of a project that has partnered with the SibYAC about the experiences of siblings of youth with a neurodisability. ${ }^{30}$ We will post the findings of this review to share with the community. Our research team, including our SibYAC partners, will seek opportunities to share both preliminary and final findings with key stakeholders such as healthcare providers, researchers, patient and family advocacy groups.

\section{Author affiliations}

${ }^{1}$ School of Rehabilitation Science and CanChild Centre for Childhood Disability Research, McMaster University, Hamilton, Ontario, Canada

${ }^{2}$ School of Nursing, Department of Health Research Methods, Evidence, and Impact, and Offord Centre for Child Studies, McMaster University, Hamilton, Ontario, Canada ${ }^{3}$ Centre of Excellence for Rehabilitation Medicine, UMC Utrecht and De Hoogstraat Rehabilitation, Utrecht, The Netherlands

${ }^{4}$ CanChild Centre for Childhood Disability Research, McMaster University, Hamilton, Ontario, Canada

${ }^{5}$ CanChild Centre for Childhood Disability Research, Department of Pediatrics and School of Rehabilitation Science, McMaster University, Hamilton, Ontario, Canada

Twitter Linda Nguyen @lindaa728, Jael Bootsma @BootsmaJael, Susan Jack @ jacksm08, Marjolijn Ketelaar @MarjolijnKetela and Jan Willem Gorter @Dr_Gorter

Acknowledgements This review will contribute towards the doctoral degree for the primary author (LN). We gratefully acknowledge our partnership with the Sibling Youth Advisory Council throughout the process of designing the protocol of this review. The authors would also like to acknowledge the assistance and guidance from Laura Banfield for the development and refinement of the search strategy.

Contributors LN, BDR, SJ, MK and JWG contributed to the conceptualisation of the review; and LN, JB, BDR, SJ, MK and JWG contributed to its design. LN drafted the manuscript, and all authors were involved in the revisions, reviewed the manuscript and provided their final approval.

Funding This review is funded by the Canadian Institutes of Health Research Patient-Oriented Research Award-Transition to Leadership Stream-Phase 1 held by LN. The partnership with the Sibling Youth Advisory Council is financially supported by the Graduate Student Fellowship in Patient-Oriented Research through the CHILD-BRIGHT Network held by LN. The Scotiabank Chair in Child Health Research is held by JWG.

Competing interests None declared.

Patient consent for publication Not required.

Provenance and peer review Not commissioned; externally peer reviewed.

Supplemental material This content has been supplied by the author(s). It has not been vetted by BMJ Publishing Group Limited (BMJ) and may not have been peer-reviewed. Any opinions or recommendations discussed are solely those of the author(s) and are not endorsed by BMJ. BMJ disclaims all liability and responsibility arising from any reliance placed on the content. Where the content includes any translated material, BMJ does not warrant the accuracy and reliability of the translations (including but not limited to local regulations, clinical guidelines, terminology, drug names and drug dosages), and is not responsible for any error and/or omissions arising from translation and adaptation or otherwise.

Open access This is an open access article distributed in accordance with the Creative Commons Attribution Non Commercial (CC BY-NC 4.0) license, which permits others to distribute, remix, adapt, build upon this work non-commercially, and license their derivative works on different terms, provided the original work is properly cited, appropriate credit is given, any changes made indicated, and the use is non-commercial. See: http://creativecommons.org/licenses/by-nc/4.0/. 


\section{ORCID iDs}

Linda Nguyen http://orcid.org/0000-0002-7165-6678

Jael Bootsma http://orcid.org/0000-0002-7862-0109

Briano Di Rezze http://orcid.org/0000-0002-6061-2506

Susan Jack http://orcid.org/0000-0003-4380-620X

Marjolijn Ketelaar http://orcid.org/0000-0002-8324-518X

Jan Willem Gorter http://orcid.org/0000-0002-3012-2119

\section{REFERENCES}

1 UNICEF. The state of the world's children 2006: excluded and invisible. New York: United Nations Children's Fund, 2005.

2 Morris C, Janssens A, Tomlinson R, et al. Towards a definition of neurodisability: a Delphi survey. Dev Med Child Neurol 2013;55:1103-8.

3 Arnett JJ. Emerging adulthood. A theory of development from the late teens through the twenties. Am Psychol 2000;55:469-80.

4 Freeman M, Stewart D, Cunningham CE, et al. Information needs of young people with cerebral palsy and their families during the transition to adulthood: a scoping review. J Transit Med 2018;1.

5 Dew A, Llewellyn G, Balandin S. Exploring the later life relationship between adults with cerebral palsy and their non-disabled siblings. Disabil Rehabil 2014;36:756-64.

6 Grossman BR, Magaña S. Introduction to the special issue: family support of persons with disabilities across the life course. J Fam Soc Work 2016;19:237-51.

7 Rosenbaum P, King S, Law M. Family-centred service. Phys Occup Ther Pediatr 1998:18:1-20.

8 Bowen M. Clinical view of the family. Fam Syst 1995;2:153-6.

9 Heller T, Kramer J. Involvement of adult siblings of persons with developmental disabilities in future planning. Intellect Dev Disabil 2009;47:208-19.

10 Corsano P, Musetti A, Guidotti L, et al. Typically developing adolescents' experience of growing up with a brother with an autism spectrum disorder. J Intellect Dev Disabil 2017;42:151-61.

11 McHale SM, Sloan J, Simeonsson RJ. Sibling relationships of children with autistic, mentally retarded, and nonhandicapped brothers and sisters. J Autism Dev Disord 1986;16:399-413.

12 McHale SM, Updegraff KA, Feinberg ME. Siblings of youth with autism spectrum disorders: theoretical perspectives on sibling relationships and individual adjustment. J Autism Dev Disord 2016;46:589-602.

13 Davys D, Haigh C. Older parents of people who have a learning disability: perceptions of future accommodation needs. $\mathrm{Br} \mathrm{J}$ Learn Disabil 2008;36:66-72.

14 Cutrona CE. Social support principles for strengthening families: messages from the USA. In: Canavan J, Dolan P, Pinkerton J, eds. Family support: direction from diversity. London: Jessica Kingsley, 2000: 103-22.
15 D'Arcy F, Flynn J, McCarthy Y, et al. Sibshops: an evaluation of an Interagency model. J Intellect Disabil 2005;9:43-57.

16 Conroy-Harris A. England and Wales. Adopt Foster 2019;43:91-4

17 Hayden NK, McCaffrey M, Fraser-Lim C, et al. Supporting siblings of children with a special educational need or disability: an evaluation of sibs talk, a one-to-one intervention delivered by staff in mainstream schools. Support Learn 2019;34:404-20.

18 Lobato DJ, Kao BT. Integrated sibling-parent group intervention to improve sibling knowledge and adjustment to chronic illness and disability. J Pediatr Psychol 2002;27:711-6.

19 Sheikh R, Patino V, Cengher M, et al. Augmenting sibling support with Parent-Sibling training in families of children with autism. Dev Neurorehabil 2019;22:542-52.

20 Redquest BK, Tint A, Ries $\mathrm{H}$, et al. Support needs of Canadian adult siblings of brothers and sisters with intellectual/developmental disabilities. J Policy Pract Intellect Disabil 2020;17:239-46.

21 Hartling L, Milne A, Tjosvold L, et al. A systematic review of interventions to support siblings of children with chronic illness or disability. J Paediatr Child Health 2014;50:E26-38.

22 Tudor ME, Lerner MD. Intervention and support for siblings of youth with developmental disabilities: a systematic review. Clin Child Fam Psychol Rev 2015;18:1-23.

23 McKenzie Smith M, Pinto Pereira S, Chan L, et al. Impact of wellbeing interventions for siblings of children and young people with a chronic physical or mental health condition: a systematic review and meta-analysis. Clin Child Fam Psychol Rev 2018;21:246-65.

24 Craig P, Dieppe P, Macintyre S, et al. Developing and evaluating complex interventions: the new medical Research Council guidance. BMJ 2008;337:a1655.

25 Peters M, Godfrey C, Mclnerney P. Scoping Reviews. In: Aromataris E, Munn Z, eds. Joanna Briggs Institute Reviewer's Manual. JBI Manual for Evidence Synthesis, 2020

26 Tricco AC, Lillie E, Zarin W, et al. PRISMA extension for scoping reviews (PRISMA-ScR): checklist and explanation. Ann Intern Med 2018;169:467-73.

27 Canadian Institutes of Health Research. Guide to knowledge translation planning at CIHR: integrated and end-of -grant approaches, 2015. Available: http://www.cihr-irsc.gc.ca/e/45321. html\#a3 [Accessed 22 Apr 2019].

28 Richardson SLL, Jordan LS. Qualitative inquiry of sibling relationships: reinforcement of disability devaluation through the exclusion of voices. Disabil Soc 2017;32:1534-54.

29 Moher D, Liberati A, Tetzlaff J, et al. Preferred reporting items for systematic reviews and meta-analyses: the PRISMA statement. PLoS Med 2009;6:e1000097.

30 CanChild Centre for Childhood Disability Research. BrothErs and Sisters involvement in health care TranSition for youth wlth Brainbased disabilitieS (BEST SIBS) Study, 2021. Available: https:// canchild.ca/en/research-in-practice/current-studies/brothers-andsisters-involvement-in-health-care-transition-for-youth-with-brainbased-disabilities-best-sibs-study [Accessed 6 May 2021]. 